\title{
Modelo de escuela de ciclismo para la Formación Integral de los ciclistas de Alto Rendimiento Deportivo en Guayaquil- Ecuador.
}

\author{
PhD Maritza Gisella Paula Chica \\ Carrera de Educación Física, Deportes y Recreación \\ Universidad Estatal Península de Santa Elena (UPSE) \\ Campus La Libertad, vía principal Santa Elena - La Libertad \\ La Libertad-Ecuador \\ m.g.pauli@gmail.com
}

\begin{abstract}
Resumen
A nivel internacional el Ciclismo se ha colocado en unos de los peldaños más relevantes. El Ciclismo deporte espectáculo se ha diversificado en sus modalidades Olímpicas. En el mundo en la formación de los Atletas del Ciclismo solo se realiza el entrenamiento deportivo, es decir se tiende a hacer al hombre y la mujer una máquina que maneja otra máquina. En los tiempos actuales están ocurriendo profundos cambios tecnológicos en el área del ciclismo. La globalización es una variable relevante a tener en cuenta como resultado inevitable del desarrollo histórico de la sociedad, sus fuerzas productivas y el desarrollo de la ciencia y la técnica. El desarrollo del deporte en Latinoamérica incluido el Ciclismo tiene que estar a tono con las tendencias innovadoras que se están desarrollando en este "Cambio de Época". El paradigma que debe regir en nuestros países es el rescate de los principios del amateurismo, adecuados a las condiciones económicas actuales y cuya base de realización debe ser un paradigma humanista. Un modelo de escuela para cualquier deporte y en particular para el ciclismo, debe contener en su concepción una triada obligatoria: la formación ciudadana, la formación cultural general integral, la formación deportivas estructuradas y coherentemente desarrolladas.
\end{abstract}

Palabras Claves: Formación integral, Escuela de Ciclismo, Modelo de Escuela

\begin{abstract}
Internationally, Cycling has been placed in one of the most important stages. Cycling, a spectacular sport has diversified its Olympic modalities. In the world the formation of cycling athletes is performed only by sports training, ie it tends to make a man and woman a machine that runs another machine. In this modern age profound technological changes are occurring in the area of cycling. Globalization is a relevant variable to be considered as an inevitable result of historical development of society, its productive forces and the development of science and technology. The development of sport in Latin America including Cycling has to be in tune with innovative trends that are developing in this "Time of change". The paradigm that should govern our countries is the rescue of the principles of amateurism, appropriate to the current economic conditions and the basis of achievement must be a humanist paradigm. A model school for any sport and in particular for cycling, must contain in its inception a mandatory triad: civic education, comprehensive general cultural training, sports training structured and consistently developed.
\end{abstract}

Keywords: comprehensive training, School of Cycling, Model School 


\section{INSTITUTO DE INVESTIGACIÓN CIENTÍFICA Y DESARROLLO TECNOLÓGICO \\ INCYT - UPSE}

\section{Introducción}

¡Alto Rendimiento Deportivo, Preparación Física, Preparación Psicológica, entrenamientos, competencias! Grandes palabras, para un atleta que se encuentra incursionando en la vida deportiva y que promueve su prestigio local, nacional o internacional para un país; nos reflejan la complejidad y trascendencia de la preparación profesional de estas futuras semillas que darán en su tiempo grandes frutos. Estas palabras enclaustran la inmensa y decisiva vida para su formación.

Los avances del deporte de Alta Competición se observan en la actualidad como una evolución de fuertes crecientes, ya que van desde las innovaciones diarias para poder llegar a unos de los rankings más altos sin importar precio absoluto. De ello el ciclismo se ha colocado como uno de los deportes más practicados por la sociedad, y donde ha hecho un ingreso muy fuerte para reunir cualidades en la salud, el medio ambiente, y a la vez su práctica deportiva.

Sin olvidar el surgimiento los Juegos en la antigüedad, comenzando con competencias deportivas en Olimpia, Grecia, aproximadamente 3000 años antes. Inspiraron los Juegos en Olimpia al desarrollo de los Juegos Panelénicos, que también incluían a los Juegos en Delhi (Juegos Píteos), Corintia (Juegos Istmitos) y Nemeas (Juegos de Nemeas). En esas épocas las guerras y disputas eran detenidas antes, durante y después de la temporada de competiciones ya que era muy importante la paz. (24)

América Latina y los países del tercer Mundo, se han visto en un proceso de cambio, que se caracterizan por su nivel de crecientes resultados y que a la vez van en múltiples direcciones, provocando en otros trascender con malas influencias a nivel deportivo. Estos incitando, en la mayoría de veces, causas disparejas en los deportes que quieren alcanzar el Alto Rendimiento y que se asocian a los casos de muy bajos desarrollo deportivo.

Ecuador se proyecta hacia el cumplimiento de este objetivo materializado con la formación integral de un deporte revolucionario de ideas y pensamientos que deben de ser cambiados, la cual responda a determinadas demandas de los países del Tercer Mundo en lo relativo a la formación de atletas integrales en el área de ciclismo, encaminados a la obtención de dicho resultado; fundamentalmente a partir de la aplicación de métodos empíricos que permitan discernir la siguiente.

Situación Problémica.

$\checkmark$ A nivel internacional el Ciclismo se ha colocado en unos de los peldaños más relevantes, sin embargo en el contexto ecuatoriano no reciben la atención suficiente para alcanzar esos niveles.
En Ecuador no se atiende lo suficiente la formación del Ciclista, tienden a convertir al hombre y la mujer en la máquina que maneja otra máquina.

$\checkmark \quad$ El paradigma humanista que acoge los principios del amateurismo, está siendo sustituido por un paradigma mercantilista.

Nuestra idea científica a defender.

Actualmente al ciclismo en Guayaquil-Ecuador hay que darle una solución a la formación integral de los Ciclistas de Alto Rendimiento Deportivo, que se vea implícita la búsqueda de una mejor preparación a Nivel Nacional e Internacional.

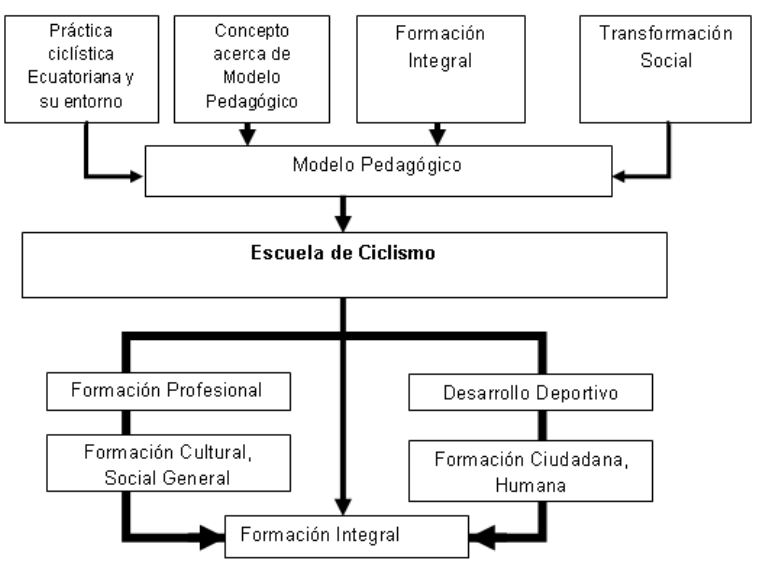

Figura 1. Formación Integral

\subsection{Acercamiento teórico al concepto de formación integral.}

Se busca en la formación integral de los atletas el desarrollo de los procesos educativos informativos, formativos en la preparación de su rendimiento deportivo. Los Educativos informativos van dirigido a las partes culturales, académicas y disciplinarias, que en este caso se traducen en los elementos de la educación teórico - práctica y metodológico que rodean a un objeto disciplinario. Los educativos formativos, se refieren al desarrollo de habilidades y a la integración de valores, que vinculen la dignidad, el patriotismo, el humanismo, la solidaridad, la honradez, y que cada uno de ellos se vincule uno con el otro. Donde se llevara el rendimiento deportivo vinculado en cada actividad que hagan con las dos antes mencionadas.

Otros autores fundamentan diferentes teorías Jesús B. Ruiz 2009 Profesor- Entrenador de la Escuela Cubana de Ciclismo y de la Escuela Internacional de Educación Física y Deporte. "La formación Integral en los atletas, no solo es el entrenamiento deportivo; en el caso nuestro de los entrenadores, es formarlos en un futuro para que representen a nuestro país en cualquier competencia, que estén revolucionariamente comprometidos para afrontar 


\section{INSTITUTO DE INVESTIGACIÓN CIENTÍFICA Y DESARROLLO TECNOLÓGICO \\ INCYT - UPSE}

cualquier situación. Es primordial la formación de los principios revolucionarios, el saber defender su patria, la revolución y nuestros principios".

Leonel Á. Díaz Jefe del área femenino - entrenador del equipo nacional femenino de la Escuela Cubana de Ciclismo "La formación integral de nuestros atletas se concibe desde dos puntos de vista. En la parte intelectual, se trata que los atletas tengan un oficio, tenga un estudio, tenga una carrera, se trata que estos atletas sean capaces de desenvolverse en su vida normal, después de su estancia en el deporte, hay que prepararlo, fortalecerlo en todos sus aspecto, también en el aspecto ideológico, saber que van a defender a la patria, el amor a su pueblo. Son importantes esos arraigos nacionales de cada país o en el país nuestro, trabajarlo en la esfera social, el trabajo en la esfera productiva, esfera de la defensa de la patria, haciendo la guardia en el momento que les toque, es un trabajo integral".

A partir de estos conceptos daremos partida al concepto de formación integral para el modelo de escuela de ciclismo de Alto Rendimiento Deportivo en Guayaquil Ecuador, lo cual se verá implícita en el concepto en la preparación práctica - teórica de los atletas en sus diferentes especialidades, y que darán paso a un surgimiento de ideas innovadoras en el ámbito actual Ecuatoriano donde el ciclista se vincule de una forma directa a la vida cotidiana con sus respectivos cambios y primicias en este cambio de Época.

La autora define como Formación Integral para el modelo de Escuela de Ciclismo de Alto Rendimiento Deportivo "No es únicamente la enseñanza y el aprendizaje del desarrollo deportivo o saberes científicos, tecnológicos es más bien una educación interdisciplinaria, humanista, social, intelectual, profesional, que transcienda a la sociedad. Es saber defender lo que uno siente, sabe ha desarrollado durante varios años, es darle al sujeto herramientas con las cuales pueda defenderse y formarse, como entrenador, masajista, psicológico, mecánico, nutricionista, entre otras". Es llevar ante las adversidades en esta nueva sociedad transportándolas a cabo en la práctica deportiva. Es una forma de preparar al sujeto para la ida y que a la vez se comprometa consigo y con la patria.

\subsection{Rasgos que caracterizan a una escuela como institución social.}

Se ha indagado sobre la historia de la escuela y su aprendizaje, según las investigaciones de los etnógrafos, parece ser que los primero vestigios de aprendizaje escolar tuvieron lugar alrededor del año 300 a.n.e en la civilización sumeria-asentada en el delta del Tigres el Eufrates-, con el nacimiento del primer sistema de escritura conocido. En unas tablillas de cera se reflejan las cuentas y transacciones agrícolas que era la forma de vida de aquella sociedad. Con la escritura nace la necesidad de formas escribas en las llamadas "casa de tablillas", las primeras escuelas de la historia de las que hay registro escrito. (25)

En la Educación Avanzada de la Escuela cubana pedagógica definen escuela como el modelo educativo genérico que se construye y revela en torno a un sistema de ideas, conceptos y representaciones sobre un objeto, fenómeno o proceso particular. Poseen un aparto categorial perfectamente organizado que guían, se revelan y se difunde (de forma espontánea y/o intencional) en la práctica social y/o por medio de la educación consciente organizada. Y desde esta posición es que la institución educativa es sinónimo de escuela. (1) (24)

En varios sitios web se encuentran diversos modelos de escuela con las distintas concepciones de la enseñanza que han existido a lo largo de la historia como lo son:

Escuela selectiva se la ha considerado como una cultura dominante y que a la vez contienen valores recalcando que son buenos por los cuales son los que deben transmitir la escuela. (8)

Escuela compensatoria, es donde existe la compensación del déficit para luego alcanzar esos valores de la cultura dominante.

Escuela comprensiva, reflexiona que todas tienen valores positivos negativos que no hay mejores ni peores que todas las culturas son diferentes. Las personas también son diferentes y hay que adaptarse a ellas, a sus ritmos de aprendizaje.

Escuela inclusiva, considera con los mismos derechos a todas las culturas y todas las personas iguales. Hay que transformar el entorno para acabar con la desigualdad. Partiendo de los contenidos de cada hombre hay que romper las barreras que la propia escuela crea a cada uno de ellos. (11).

Las escuelas de ciclismo a nivel mundial han comenzado desde el año 1982 con la finalidad de preparar al ciclista físicamente y como prueba tenemos la escuela en manos de José Miguel Carrero, como Director de la misma en España, y de Adolfo Moreno Pérez, como Director Técnico. Escuelas de Ciclismo que han pasado centenas de corredores, todos con la finalidad de formarlos en el mundo de la carrera y que han hecho su debut en el campo Elite y Sub 23. Además en estos 30 años muchos de ellos dieron alegrías a ese club, llegando al campo profesional como es el caso de Joan Valbuena Roset (Lotus-Festina), Antonio González (Transmallorca- 


\section{INSTITUTO DE INVESTIGACIÓN CIENTÍFICA Y DESARROLLO TECNOLÓGICO INCYT - UPSE}

Gios y Colchó, CR) y más recientemente Israel Núñez (ASC y Kaiku).

Observamos que en los proyecto de Escuelas de Ciclismo como principal objetivo tienen la formación deportiva, humana y social de los jóvenes ciclistas, sin ningún tipo de distinción de sexo o discapacidad. Donde se proponen aspiraciones en su proyección en una nueva y amplia dimensión (local, regional y autonómico), en estrecha colaboración con el Patronat Municipal d'Esports de Sant Boi de Llobregat, para mejorar el nivel técnico y competitivo de los ciclistas olvidando su formación integral para luego vincular a estos ciclistas a la sociedad actual. (6)

\section{Metodología y caracterización de los resultados empleados en los ciclistas nacionales y extranjeros.}

En este apartado se concibe lo concerniente al proceso y desarrollado de las indagaciones realizadas a los atletas, entrenadores masajista, sponsores, dirigentes, que se encuentran vinculados a la familia ciclística mundial. Para obtener la información necesaria, llevándola de tal forma que con su análisis se puedan establecer bases teóricas, metodológicas y prácticas. Teniendo como fuentes primarias los documentos oficiales de las respectivas indagaciones realizadas a los atletas extranjeros y nacionales, arrojaron sus respectivas resultados dando paso al diseño investigativo que la autora propone en el trabajo.

\subsection{Metodología empleada.}

El siguiente trabajo científico e investigativo se realiza con los atletas y dirigentes del ciclismo con una muestra de 84 atletas de 13 países del mundo tales como (Italia, Ucraina, Moldava, Venezuela, España, Ecuador, Portugal, Austria, México, Canadá, Panamá, Alemania y Cuba. Fueron entrevistados 18 dirigentes de diez países como: Italia, Panamá, Venezuela, Ecuador, España, Australia, México, Canadá, Alemania y Cuba. Para la realización del diagnostico se utilizaron diferentes métodos y técnicas de investigación que nos permitió revelar lo que está sucediendo en la actualidad de ciclismo ecuatoriano, a la vez relacionarlo, pudiendo pasar de lo general a lo especifico y viceversa sin duda asumir una crítica respectiva de la información recolectada y poder dar un criterio valorativo de la verdad fundamentando en su concepción de una escuela de ciclismo.

Tabla 1. Tabla ejemplo

\begin{tabular}{|l|l|c|}
\hline \multirow{2}{*}{$N^{\circ}$} & \multicolumn{2}{|l|}{ Atletas que participaron en la encuesta: } \\
\cline { 2 - 3 } & Países & Atletas \\
\hline
\end{tabular}

\begin{tabular}{|r|l|c|}
\cline { 2 - 3 } 1 & Italia & 7 \\
\hline 2 & Ucraina & 7 \\
\hline 3 & Moldava & 7 \\
\hline 4 & Venezuela & 7 \\
\hline 5 & España & 7 \\
\hline 6 & Ecuador & 4 \\
\hline 7 & Portugal & 5 \\
\hline 8 & Austria & 6 \\
\hline 9 & Cuba & 6 \\
\hline 10 & México & 7 \\
\hline 11 & Canadá & 6 \\
\hline 12 & Panamá & 7 \\
\hline 13 & Alemania & 8 \\
\hline & TOTAL & 84 \\
\hline
\end{tabular}

Reconociendo la importancia del trabajo y valorando las indagaciones realizadas, pasaremos al análisis de las fases investigativas, interviniendo en la correlación para el desarrollo de las siguientes fases que se expone en la tesis así poder concebir sus resultados. En la parte principal de este estudio debemos tener implícito unas series de guía tanto generales como específicos para tener en cuenta la realización de este trabajo investigativo.

Cada día se impone en el mundo actual, el compromiso de dar amplitud a las diferentes fases de la investigación, dada la necesidad de elevar el nivel del trabajo científico y poder llevar a cabo las fases que se interrelacionan lógicamente en el diagnóstico efectuado a los ciclistas y dirigentes por diferentes métodos donde constituye el fundamento de la escuela de ciclismo en la ciudad de Guayaquil- Ecuador.

Por lo expresado anteriormente podemos valorar rápidamente la precisión del problema planteado como contribuir a la formación integral de los atletas en la escuela de ciclismo. Formando parte indispensable para la solución del mismo debemos valorar entonces la base de todas las transformaciones personales de los atletas para su preparación profesional. (3)

Entrando en la parte principal de este estudio debemos plantear que la formación integral lleva implícito una serie de valores tanto generales como específicos los cuales debemos tener en cuenta para el desarrollo del modelo de escuela de ciclismo que proponemos.

Las tendencias y reflexiones recientes de la investigación sobre la formación integral han enfatizado en su dimensión cuantitativa y cualitativa considerando esos factores determinantes de la relación entre esas dimensiones podemos precisar el estudio teórico y empírico realizado en esta investigación. En ese sentido el problema de la investigación es dar solución a la formación integral de los ciclistas de alto rendimiento. Hay que recordar que una actividad consustancial a la propia existencia 


\section{INSTITUTO DE INVESTIGACIÓN CIENTÍFICA Y DESARROLLO TECNOLÓGICO \\ INCYT - UPSE}

del hombre como un ente social, está encargada en desarrollar la naturaleza, y las propias ideas del ser humano, preparando al hombre en el aspecto social, humano, profesional, para soportar los embates del desarrollo y que este no le afecte en su vida cotidiana. (31)

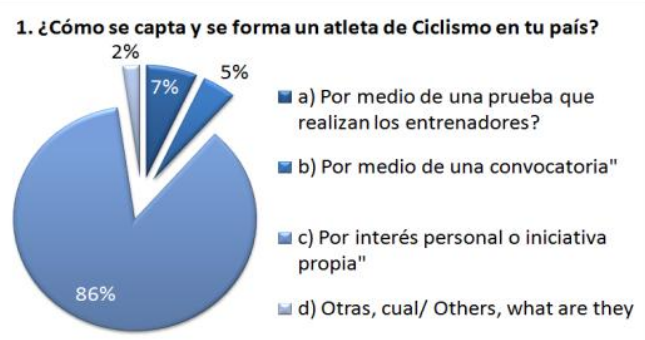

Figura 2. ¿Cómo se capta y se forma a un atleta de Ciclismo en tu país?

2. ¿Cómo caracterizas a las escuelas de ciclismo en tu país?

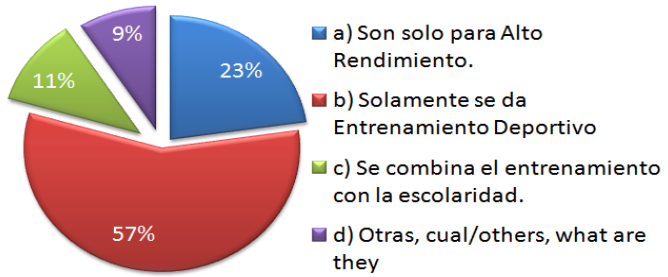

Figura 3. ¿Cómo caracterizas a las escuelas de ciclismo en tu país?

3. ¿Que ventajas tiene la escuela de Ciclismo en tu país?

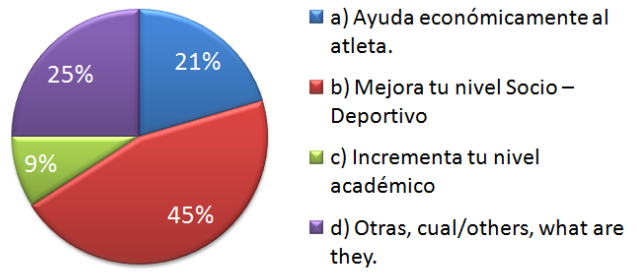

Figura 4. ¿Qué ventajas tiene la escuela de Ciclismo en tu país?

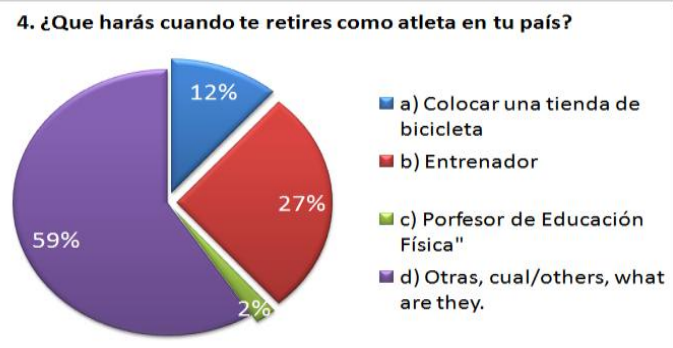

Figura 5. ¿Qué harás cuando te retires como atleta en tu país?

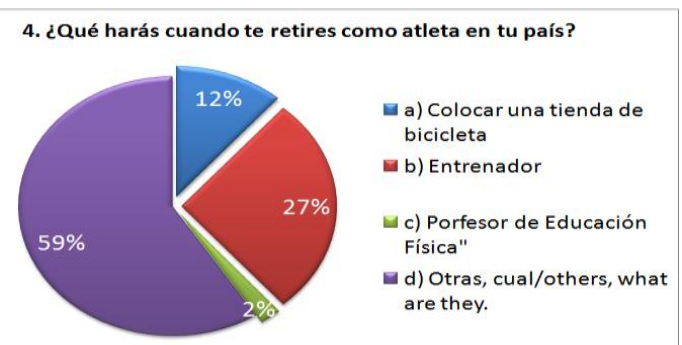

Figura 6. ¿Qué harás cuando te retires como atleta en tu país?

5. ¿Cómo te gustaría la escuela de Ciclismo en la que tu entrenas?
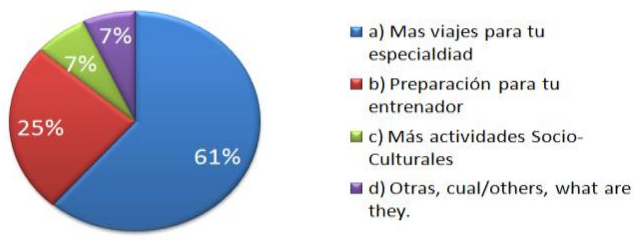

Figura 7. ¿Cómo te gustaría la escuela de Ciclismo en la que tú entrenas?

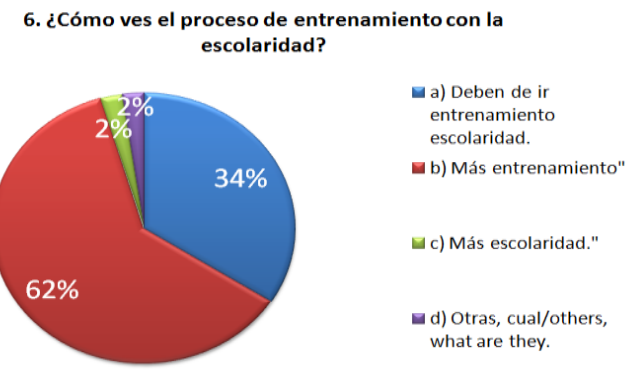

Figura 8. ¿Cómo ves el proceso de entrenamiento con la escolaridad?

El resultado del diagnostico va dirigido ante todo al estudio de cada indagación realizada, arrojando valores importantes para el desarrollo del cuerpo de la investigación. Naturalmente, la solución de este está en preparar al atleta y en la construcción de un modelo de escuela que permita una sociedad de atletas con una formación integral, multilateral y armónica desarrollada, el cual pueda este deportista dar respuesta a las necesidades que esta nueva sociedad demanda día a día y a la vez contribuir a su propio desarrollo profesional. Como parte esencial de la tesis el diagnostico contribuye de forma determinante a los diferentes procesos de formación integral alcanzado en el deporte de alto rendimiento un nivel preciso para su preparación.

La vida cotidiana y el Deporte, no constituyen en nuestra sociedad factores opuestos, sino que representan partes diferentes de un proceso integrado del perfeccionamiento de la formación integral de los ciclistas. Esta integración se fundamenta por una concepción teórica del desarrollo de la personalidad y 


\section{INSTITUTO DE INVESTIGACIÓN CIENTÍFICA Y DESARROLLO TECNOLÓGICO \\ INCYT - UPSE}

por otra parte en las diferentes concepciones concretas al desarrollo de nuestra nación, estos cambios en el hombre alcanzan una mayor significación, por su repercusión en el continuo desarrollo de la preparación social de estos atletas.

La revisión bibliográfica, el estudio y análisis de documentos, los estudios realizados sobre las diferentes escuelas de ciclismo, la implantación de los métodos y técnicas investigativas como validación de tesis de maestría, doctorados y trabajos de diplomas de maestrantes, aspirantes y estudiantes sobre el desarrollo y comportamiento de estas instituciones produjeron resultados objetivos de los instrumentos aplicados.

Estos se basaron en temáticas de estudio y perfeccionamiento de las escuelas deportivas, donde a la vez posibilitó elaborar la fundamentación teórica y metodológica del modelo de escuela de ciclismo, con un enfoque sistémico y donde se revela como médula del proceso, el funcionamiento de la institución deportiva, con sus indicadores, principios, barreras, funciones y otros aspectos. (5)

En aras de lograr las temáticas debemos de partir cuales son las primicias que dan lugar a la práctica sistemática de las escuelas de ciclismo a través de la fundamentación y elaboración de la propuesta. También se concibió la representación gráfica del modelo, así como su funcionamiento y operacionalidad, para poder darle seguimiento a la misma desde las diferentes perspectivas de estudio plasmadas.

Para la implantación y validación del modelo, se tuvo en cuenta diferentes fases (selección de la muestra, aplicación de instrumentos de investigación tales como encuestas y entrevistas, análisis de resultados y ajustes de modelo); demás, para su validación de la efectividad se definieron indicadores los cuales se midieron mediante encuestas, entrevistas, observaciones y aplicación de procedimientos estadísticos, y a la vez darle seguimiento nacional e internacional en sus diferentes dimensiones.

En esa misma medida va tomando importancia la realización del modelo de escuela de ciclismo para la formación integral de los ciclistas de alto rendimiento la solución es preparar al hombre para su vida profesional.

La inversión que se hace en la implementación y validación del modelo de escuela de ciclismo. Repercute de una forma positiva no solo en el aspecto de la salud, donde va un aumento progresivo de las cargas o un incremento de las capacidades biomotoras del atleta tales como (fuerza, resistencia, entre otras) sino que a la vez producen un mayor impacto en la sociedad en que ellos se relacionan, lo que al mismo tiempo, propicia la elevación de la preparación del atleta a la sociedad actual. Permitiendo un avance no solo físico sino intelectual, humanista, y profesional. (12)

\section{Diseño de un modelo de escuela de ciclismo para la formación integral de los ciclistas de alto rendimiento deportivo en Guayaquil-Ecuador}

\subsection{Proceso de Modelación}

La formación integral de los ciclistas va dirigida al cumplimiento del fin del modelo de escuela de ciclismo, que se manifiesta para el deporte en función de la preparación óptima de los atletas, en un determinado ámbito deportivo, para así facilitar a la escuela el cumplimiento de sus objetivos, y propósito en el desempeño de un ciclista integral.

El presente modelo que se fundamenta en la investigación, se observa la estructuración de una escuela de ciclismo para la formación integral, mediante un estudio realizado, que posibilitará mayor sistematización en el proceso de formación integral en los ciclistas en Guayaquil Ecuador. El proceso de modelación de la escuela se produce bajo un diverso sistema de influencias positivas y negativas, donde el ciclista debe de enfrenar y vencer con la preparación de un grupo de profesionales del área deportiva, donde a la vez el atleta debe asumir el reto de dirigir el proceso docente-educativo con la práctica ciclística desde la llegada al centro. (17)

Cumplir con este propósito requiere de un proceso de auto preparación permanente no solo de los ciclistas sino de los dirigentes que llevan a cabo este proceso de transformación, basado en una solida motivación profesional que movilice todos los esfuerzos de todos los que componen la escuela de ciclismo al cumplimiento de las tareas y funciones que se plantean.

El dirigente que toma parte principal de este proceso como promotor de una cultura general integral, refuerza su misión de portador de las interrelaciones de la escuela con las diferentes disciplinas del ciclismo con la sociedad y la familia, coordinando el sistema de acciones educativas y prácticas del ciclismo, que potencian su influencia a favor del cumplimiento de los objetivos generales del sistema educativo en el logro del desarrollo de la preparación de los ciclistas. (14)

El profesional de la enseñanza del ciclismo constituye una figura emblemática para el ciclista por la influencia educativa en el logro del desarrollo de su preparación profesional. Es necesario entonces, una formación integral que transite desde los elementos más generales del devenir de la practica ciclística 


\section{INSTITUTO DE INVESTIGACIÓN CIENTÍFICA Y DESARROLLO TECNOLÓGICO \\ INCYT - UPSE}

hasta la actualidad, para compensar las insuficiencias espirituales del sujeto desde su propio medio, logrando la innovación del entorno social desde un punto de vista creativa y desarrolladora que concibe el trabajo del profesional cada vez mejor llevándolo hacia la actividad de forma colectiva, influenciadas cada una, en el trabajo educativo desde sus potencialidades, como sujeto idóneo de transferir prudencias, experiencias y valores a los demás.

El hecho de que se investigue, sugiera y proponga un modelo de escuela de ciclismo significa alimentar la tesis en la formación integral de los ciclistas, ya que esta juega un papel de dirección y conducción de la preparación de cada uno de ellos.

\subsection{Referentes Teóricos asumidos por Autores}

La sociedad, la naturaleza y el pensamiento del ser humano establecen sistemas multidisciplinarios de excepcional complejidad, en la elaboración de los modelos, los que deben de tener en cuenta para su indestructible planificación, transformación y desarrollo, donde se vean implícitas la influencia de un modelo sobre otros.

Los modelos se ven muy relacionados a objetos, hechos, procesos, así como figuras o personalidades que han dado un carácter más significativo a los modelos por diferentes aspectos de la realidad, donde nos hemos visto obligados a fundamentar el desarrollo histórico de la problemática que se investiga, sin dejar a un lado el carácter relativo y condicionado por las disímiles particularidades de los diseños de modelo. En el trabajo de investigación se puede apreciar referentes teóricos de modelos y a la vez pictografías encontradas en las cuevas, en la comunidad primitiva, hasta en los sofisticados ordenadores electrónicos.

Los modelo en la ciencia, continúan hasta la actualidad en una disertación en su importe explicativo, donde continúan siendo representaciones pictóricas, diseñadas por el hombre donde se les ha permitido representar, manifestar, pronosticar el entorno de la sociedad, atendiendo a la multiplicidad, de las variedades y el desarrollo continuo de la misma. En el área de la rama científica poseen los modelos sus particularidades, donde los investigadores asumen heterogéneas definiciones o clasificación de modelos en correspondencia del tipo de ciencia que se trate o de los objetivos que se representen. Donde a la vez surgen las ideas sobre los modelos como lo es el diseño curricular, los modelos para elaborar una estrategia, los de diagnostico, y así muchos hasta que aparecieron investigaciones con Carlos Álvarez, Victoria Chirino, A. Marimón, Fernando González Rey, Fátima Addine, y Nerelys de Armas. Otros trabajos encontrados están los de L. García, 1998; B.
Castellanos, 2005; Ariel Ruiz, 2005; F. González, 1999; teniendo en cuenta que estos trabajos ellos no lo asumen como objetivos.

La escuela de ciclismo parte de la búsqueda de modelos porque estamos en presencia de una teoría pedagógica o parte de ella, que a la vez nos impulsó a idear algunas representaciones que nos podrían ayudar para perfilar criterios al momento de la elaboración del presente trabajo. La actual investigación y valoración parte de una determinada base como el de "modelos" donde nos llevo a la indagación del mismo.

En el manejo de los modelos la investigación pedagógica se coloca en un lugar cada vez más importante, ya que con ellos se transforma en medio y método para las investigaciones de carácter científico pedagógico. Nos vemos comprometidos en la investigación y en la instrucción de este término "modelo" ya que adquiere una relevancia por su forma única en la representación de trabajos ya que exige determinados requerimientos y funciones.

En la búsqueda de información acerca de la palabra modelo proviene del latín modulus que significa medida, ritmo, magnitud, y está relacionada con la palabra modus que significa copia, imagen. Manifiesta Ordaz Lorenzo R. que la primera la denominan Preteórica la cual permaneció muchos siglos en la cual fueron utilizados en el arte. En la segunda manifiesta características esenciales uno se puede obtener la característica esencial del otro. La tercera etapa hasta la actualidad la reconocen como modelación como método del conocimiento teórico.

Tomamos encuenta la definición de modelos por varios autores: Según V. V. Davidov apunta que "los modelos son una forma peculiar de abstracción en la que las relaciones esenciales del objeto están fijadas en enlaces y relaciones visualmente perceptibles y representadas por elementos materiales o semióticos. Se trata de una peculiar unidad de lo singular y lo general en la que en primer plano se presenta lo general, lo esencial".(18)

Para V. Stoff el modelo es concebido mentalmente o realizado en forma material, ya que reflejando o reproduciendo el objeto de la investigación es capaz de sustituirlo de modo que su estudio nos dé una nueva información sobre dicho objeto." (28)

Esta definición es a nuestro juicio más completa que las anteriores. En ella se sostiene que el modelo es un sistema concebido mentalmente, ya que cuando se da la disyuntiva o realizado en forma material, esto presupone necesariamente una concepción mental anterior a la materialización.

Teniendo en cuenta estos análisis para nosotros el modelo científico es "la representación de aquellas características esenciales del objeto que se investiga, 


\section{INSTITUTO DE INVESTIGACIÓN CIENTÍFICA Y DESARROLLO TECNOLÓGICO \\ INCYT - UPSE}

que cumple una función heurística, ya que permite descubrir y estudiar nuevas relaciones y cualidades de ese objeto de estudio con vistas a la transformación de la realidad" (21).

La abstracción recoge las características esenciales de un objeto ya que puede representar la materialización de un modelo.

En las ciencias pedagógicas y sobre todo en la metodología de la investigación que se ocupa de analizar el proceso pedagógico se encuentran diferentes denominaciones y tipos de modelos. Hay autores (Sierra Salcedo, A., 2002; Valera Alfonso, O., 2000; De Armas, N., 2003) han llevado a la formulación de sus propios criterios mediante la lectura y revisión de tesis de maestría y Doctorados. Sobre esos trabajos J. A. Marimón expresa: "Como se puede observar las definiciones anteriormente planteadas tienen las mismas limitaciones que muchas veces presentan los modelos, al no expresar con claridad suficiente la esencia de su objeto, por lo que recomendamos que al definir el modelo utilizado en la investigación se defina a un nivel más particular y específico enfatizando en su objeto de estudio y contextualizando su campo de acción." (29)

Desde este punto de vista, se hace necesario entonces, asumir cuáles son las características más importantes que los definen. En los procesos, existen determinadas regularidades más generales que pueden ser consideradas como principios, ellos deben ser parte esencial del modelo. De igual forma el modelo que se propone está dirigido a un fin y en este sentido debe alcanzar determinados objetivos los cuales deben formar parte del mismo.

El modelo como punto de partida para la transformación de la realidad debe ser asumido también como un conjunto de acciones, distribuidas en etapas y enmarcadas en una estrategia de trabajo.(2)

El modelo constituye un nuevo enfoque para comprender como la escuela de ciclismo se constituye como productora y reproductora de las disposiciones objetivas y subjetivas de la preparación de ciclistas en el fortalecimiento de su identidad, insertada en el concepto de desarrollo de una cultura de formación integral en el ciudadano ecuatoriano.

Constantemente se crean nuevos modelos y se modifican o destruyen otros; donde a la vez un mismo modelo puede irradiar varios objetos o fenómenos, a través de diferentes modelos, implícitamente disímiles entre sí; en su proceso de perfeccionamiento hemos concebido diferentes formaciones de modelos, por autores de reconocida transcendencia, en el área investigativa. (4)

En cortas palabras con respecto a la definición de modelo que viene del Italiano "modello" donde quiere decir representación de algo que se debe seguir o omitir. (Bringas, J.1999).

Otra definición más general nos ofrece la MsC. Irma Nocedo al señalar que "El modelo es un representación simplificada de la realidad, que cumple una función heurística, ya que permite descubrir y estudiar nuevas relacionadas y cualidades del objeto de estudio."(Nocedo, I.1989, 88), por lo cual los modelos tendrán un representación referente, que a la vez pudiera ser mejorado, prolongado, modificado y reiterado. Establece una inmediación al entorno, pero no son el entorno, sino un epígrafe de ella.

Por Ariel Ruiz 1998. Un modelo surge del estudio y por tanto no constituye una copia original del mismo. El investigador describe y representa hasta un determinado grado, la estructura, el funcionamiento y el estado del objeto. El modelo constituye una representación simplificada de la realidad que cumple una función heurística, al permitir descubrir y estudiar nuevas relaciones y cualidades del objeto estudiado y debe revelar, a la vez la naturaleza del objeto que se modela y las posibilidades teóricas metodológicas que faciliten su instrumento. (27)

Una definición por el Dr. Lizardo García donde determina que "El modelo se expresa como un grupo de formulaciones interrelacionadas, pero con independencia suficiente como para que cada una pueda expresar un contenido propio que puede ser enriquecido y concretado" (García, L.2000, 294)

En la variada literatura impresa como digital hemos consultado sobre modelos pedagógicos, donde encontramos la definición por la MsC. Regla Sierra que lo representa como una "construcción teórica formal que fundamentada científicamente e ideológicamente interpreta, diseña y ajusta la realidad pedagógica que responde a una necesidad histórica concreta." Sierra, 2002 (29).

Justamente, el modelo de escuela que se exterioriza se somete a una insuficiencia social contemporánea, donde a la vez implica el mejoramiento de la identidad nacional en el progreso de la preparación del ciudadano ecuatoriano.

La autora partió de la definición general integradora de modelo brindada por Cañal, P. y Porlan, R. Los que mencionando a J. Gimeno marcan que "un modelo no es más que una representación parcial y selectiva de aspectos de la realidad, centrando la atención, tan solo, en aquello que se considera importante. El modelo es un esquema mediador entre la realidad y el pensamiento una estructura en torno a la que organizar el conocimiento, una fuente de hipótesis de investigación, que no puede agotar de forma absoluta y definitiva la interpretación de la realidad, sino que tendrá siempre un carácter provisional y aproximativo" (20) 


\section{INSTITUTO DE INVESTIGACIÓN CIENTÍFICA Y DESARROLLO TECNOLÓGICO \\ INCYT - UPSE}

Un modelo científico nos ha llevado, que no es más que un procedimiento metodológico del conocimiento científico, un instrumento que ha permitido una investigación de carácter material o teórico creado por los científicos que permite transcribir el objeto o fenómeno a experimentar y como se señaló con anterioridad, establece similitudes con elementos conocidos previamente o diseña estructuras matemáticas o de otra índole que expliquen satisfactoriamente lo estudiado. (Pérez, G. Nocedo, I.1989)(González, V. 1990) (7) (15)

En las investigaciones realizadas por Científicos en el área de modelo encontramos en varios países como lo conciben uno de ellos en cuba se han confeccionado diferentes modelos pedagógicos para su mediación en la práctica entre ellos podemos citar: El Modelo de Escuela Cubana (García L. 1997), También encontramos El de Escuela Politécnica Cubana (Patino, M.DEL R, 1996), vinculados al estudio está, el de integración del estudio con el trabajo en la Escuela Cubana (García, G.S/F). (16) Es importante resaltar que dentro de esta documentación se han observado en el ámbito deportivo el libro de Escuela Cubana de Boxeo por parte de Alcides Segarra en el 2006. Donde ponen énfasis en la preparación del atleta, para su formación integral y en el que a la vez es uno de los deportes que más medallas ha alcanzado a nivel internacional.

Entrando un poco más al ámbito mundial hemos encontrados otros documentos que manifiestan investigaciones sobre los modelos utilizado en las transformaciones educativas en los países de América Latina, Europa y Estados Unidos. Analizamos lo que Tedesco, J.C. proponen para poder llegar al éxitos y poder abrir pasos en el contorno de la política educativa poniendo de manifiesto lo siguiente (19)

$\oplus$ Resalta en la continuidad de la política de profundos cambios

† Colocando escases de capacidad de anticipación a demandas y problemas

\$ Sobresale la información en las tendencias mundiales.

† Se distingue dispositivos de evaluación.

$\oplus$ Se destaca en el diagnóstico enfocado a la realidad presente.

$\oplus$ Culmina en la concentración de estrategias obteniendo resultados homogéneos.

En esta investigación se refleja el carácter de búsqueda en los modelos, encontrando una literatura muy variada donde, Schiefelbein, Ernesto realiza una investigación en los países de América latina llegando al éxito de los modelos educativos: (13)

๑ La reforma educativa tiene que beneficiar a todos los usuarios.
๑ Cada niño hay que garantizarle una canasta básica de materiales educativos.

$\oplus$ Realizar autogestión para niños de escasos recursos.

$\oplus$ Renovar y capacitar la didáctica de los instructores.

$\oplus$ Realizar estudios investigativos mejorando el empirismo.

๑ Tener un mejor manejo de los recursos financieros y de la reforma educativa.

๑ Dominar la Política educativas sustentada en buena información y buen análisis.

\& El Ministerio de Educación debe jugar un papel clave en la reforma educativa y elevar su calidad.

Examinando la documentación obtenida, hemos pasado a un punto muy importante donde la autora, caracteriza "Modelo de Escuela de Ciclismo", como institución social de los alumnos-atletas, profesoresentrenadores, trabajadores y dirigentes donde a la vez forman un sistema influenciado por características y funciones que permite interactuar en el proceso pedagógico, de la familia, la comunidad, pudiendo alcanzar el desempeño de fines educativos deportivos en un contorno determinado a sus condiciones y espacio Territorial. Logrando un avance científico en la preparación de los ciclistas en el área cognitiva y física en los Ecuatorianos.

\subsection{El modelo de escuela}

La educación en Ecuador está dando oportunidades a sectores poblacionales de escasos recursos económicas para una mejor educación, gracias al Gobierno del Presidente Rafael Correa. Donde la Educación, en Ecuador va "inspirada en principios éticos, pluralistas, democráticos, humanistas y científicos, promoverá el respeto a los derechos humanos, desarrollará un pensamiento crítico, fomentará el civismo; proporcionará destrezas para la eficiencia en el trabajo y la producción; que estimulará la creatividad y el pleno desarrollo de la personalidad y las especiales habilidades de cada persona; impulsará la interculturalidad, la solidaridad y la paz" (9). Sin dejar escapar que nuestro trabajo no está ajeno a esos principios, que ayudan al mejoramiento de un pueblo digno, soberano, dirigido hacia una Patria Libre y Soberana.

Nuestro modelo se debe regir a la Universalización de la Educación General Básica de primero a décimo año, constituida por el Ministerio de Educación y Cultura Ecuatoriana que a la vez permite "Brindar educación de calidad con enfoque inclusivo y de equidad, a todos los niños y niñas, para que desarrollen sus competencias de manera integral y se conviertan en ciudadanos positivos, activos, capaces 


\section{INSTITUTO DE INVESTIGACIÓN CIENTÍFICA Y DESARROLLO TECNOLÓGICO \\ INCYT - UPSE}

de preservar el medio natural y cultural y de sentirse orgullosos de su identidad pluricultural y multiétnica". (9)

Para la elaboración de nuestro modelo indagamos que "las universidades y escuelas politécnicas públicas y particulares serán personas jurídicas autónomas sin fines de lucro, que se regirán por la ley y por sus estatutos, aprobados por el Consejo Nacional de Educación Superior".

Artículos a tomar en cuenta por el Ministerio de Educación y Cultura para nuestra escuela de Ciclismo. "Art. 207.- Para la administración de los establecimientos educativos del país, facúltase al Ministro de Educación y Cultura reglamentar de la manera más adecuada los calendarios de clase, etapas de estudio, días de descanso y períodos de exámenes de la educación escolarizada, de acuerdo a las reales necesidades de la comunidad educativa y al mejor aprovechamiento de los recursos humanos, físicos y tecnológicos".

La autora para poder diseñar la escuela y el modelo conveniente partió de los criterios, reglamentos, leyes y atributos que se necesita para el mismo, tanto como centro transformador educativo para la vinculación de niños y jóvenes donde la escuela dirige el proceso de educación por intermedio de los profesores, entrenadores, personal interno, llevándolo hacia una política de autodesarrollo, adyacente a la familia, la comunidad, las organizaciones sociales estudiantiles y comunitarias entre otras.

A continuación se muestra y describe un esquema en el que se muestra el Modelo de Escuela de Ciclismo actuante en otros países del mundo y su relación con el modelo de Escuela de Ciclismo propuesto para la Formación Integral de los Ciclistas de Alto Rendimiento en Guayaquil - Ecuador.

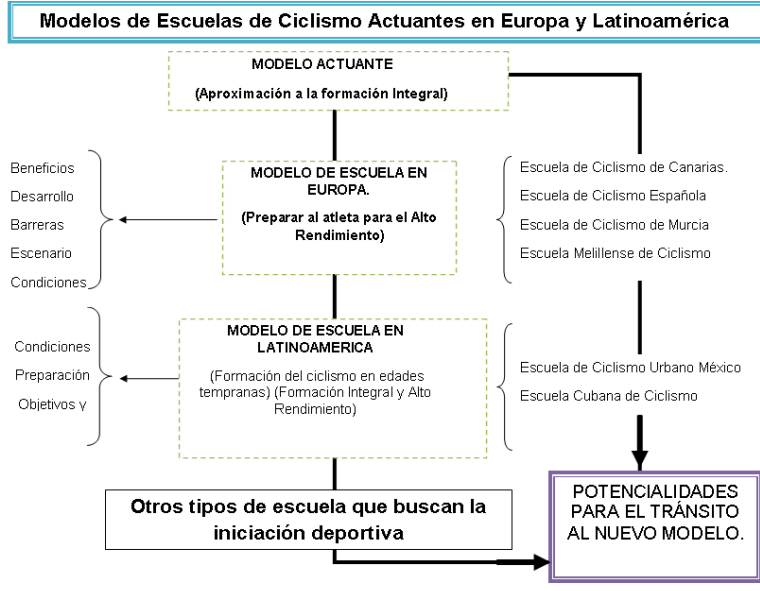

Figura 2. Modelo de Escuelas de ciclismo Actuantes.
Se muestra que el modelo de escuelas de ciclismo actuante, es a partir de un análisis de las características propias de su población y de sus recursos tanto, económico como financieros en sus Beneficios, en su desarrollo tecnológico, en sus barreras, en la estructura de sus escenarios y en las condiciones en las que estas se desenvuelven. (22) Nuestro trabajo de investigación nos va permitir la proyección del modelo de escuela de ciclismo, sobre las bases de las escuelas modeladas, donde vamos acceder en hechos más reales de nuestra población, mediante caracteres adecuados a su funcionamiento y mas no en una proyección de descripción empírica, o de ideas no adecuadas a su funcionamiento en el logro de la formación integral de los Ciclistas de Alto Rendimiento en Guayaquil Ecuador.

\section{Resultados}

La autora modela, la proyección de la escuela de ciclismo mediante un trabajo metodológico y de dirección, sin dejar atrás las escuelas modelas, donde a la vez nos permite modelar nuestro trabajo sobre las escuelas de ciclismo existentes a nivel mundial. En dependencia de cómo se adopte nuestro modelo, se puede obtener mayor o menor resultado en la formación integral de los ciclistas de Alto Rendimiento. Nuestro modelo proyectivo servirá como guía en el proceso de cambio que actualmente se está viviendo en el Ecuador con una Revolución Ciudadana que hace más de cinco décadas estaba oprimida y que permitirá por medio de este modelo ese cambio que tanto se espera.

Se tomaron en cuenta para la elaboración de nuestro modelo proyectivo la metodología utilizada en los modelos actuantes, no solo porque se reflejan características de la escuela real, sino porque nos permitió expresar y revelar las potencialidades que nuestro país tiene como transformador en la esfera educativa- deportiva, donde podemos explotarlas para su mayor auge. Asimismo, en la modelación de la escuela de ciclismo proyectiva el claustro de profesores y el cuerpo de entrenadores ocupan en el organigrama una posición privilegiada que condiciona sus posibilidades para convertirse en motor impulsor de los cambios de la potencialización y transformación de los Atletas, los padres, la sociedad y la dirección de la escuela. (23)

Teniendo en consideración que la escuela de ciclismo debe alcanzar un mayor protagonismo en el área docente, deportiva, social entre otras, en el cual la institución deba convertirse en promotor de una escuela educativa deportiva de la sociedad ecuatoriana. Este proceso puede ser catalogado de transformador en el ecuador y en el mundo ya que 


\section{INSTITUTO DE INVESTIGACIÓN CIENTÍFICA Y DESARROLLO TECNOLÓGICO \\ INCYT - UPSE}

persigue en su esencia, el cambio de una educación más integral con la preparación de un ciclista formado no solo físicamente sino que intelectualmente también, donde posibilita la interacción de los atletas.

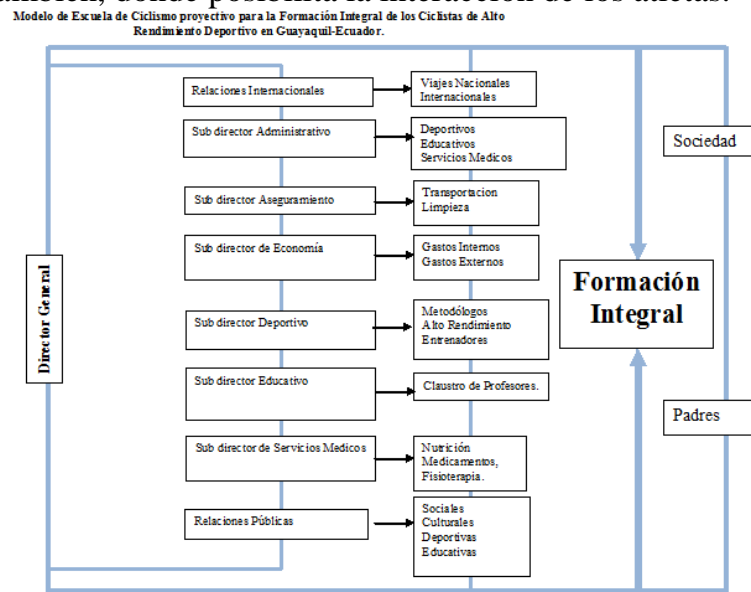

Figura 3. Esquema del modelo proyectivo de la escuela de ciclismo

Analizando todo este proceso debemos de ir involucrando poco a poco a todos los docentes y a los integrantes de este proceso en la transformación de la realidad educativa ecuatoriana, que a la vez nos permita el modelo de transformación de la escuela con sus características pertinentes donde se nos diferencie de las otras escuelas existentes, ya que no solo varían las condiciones educativas sino las condiciones deportivas que los va a permitir ser mas integral y de hacerlo un hombre útil para la sociedad en sus diferentes esferas. (10)

Actualmente, se vela que todo procedimiento de predominios educativas, en las que son fuentes principales de un desarrollo como lo es la escuela, la familia y la sociedad interactúen de una manera más lógica sin llegar a transmitir mecánicamente a los niños, las tradiciones laborales, políticas, sociales entre otras; sin llevar a las condiciones de vida ya dispuestos por los padres en su quehacer diario.

Nosotros debemos ser capaces en la formación de valores acorde con la Revolución ciudadana actual que el presidente Rafael Correa se propone ejerciendo influencias positivas en cada escuela, en las clases y en cada entrenador, donde podamos caracterizar y comprender mejor las individualidades de cada atleta y que ellos se sientan arte de este cambio histórico que vive la patria.

\section{Conclusiones}

El Modelo de Escuela que se presenta en esta investigación se fundamenta en una aproximación de una institución tan compleja como lo es un centro de
Alto Rendimiento y un centro Educativo. En el cual las líneas a seguir sobre el funcionamiento y funciones de la escuela de ciclismo en sus sistemas de relación lleven consigo un auge de mayor amplitud en cada objetivo a perseguir, que a la vez se esperan para los próximos años de su avance, en el marco histórico social previsible.

Este modelo es ajustable a las disímiles escuelas de ciclismo que se encuentran a nivel mundial, dado su carácter universal. En la actualidad, la práctica del ciclismo se observa solo el entrenamiento deportivo, concibiendo el aislamiento de una formación integral el proceso de concreción e introducción del resultado de nuestra investigación se proyecta ya para el cumplimiento de una escuela de ciclistas para su formación Integral. (26)

Por primera vez se dispone de una formación para los ciclistas la cual puede ser tomado en cuenta para otros atletas buscando el proceso de perfeccionamiento en el plano de la formación integral de los ciclistas en la escuela. Que a la vez permita contribuir a formar un atleta con un sentido de la identidad nacional, la dignidad y solidaridad humana, cuyos sentimientos acerca de la defensa de la Patria Libre y Soberana en el patriotismo, de nuestro pueblo, sus tradiciones, la preservación de la independencia y la soberanía.

Conjuntamente, al momento de la elaboración del modelo de la escuela de ciclismo permitió con claridad las peculiaridades de una escuela de ciclismo donde a la vez se tomaron como muestra otros modelos. Lo cual nos cedió propiciar procesos de apropiación características que ayuden a la formación científico-técnica, humanista y práctica acorde a las influencias sociales, grupales e individuales de los atletas que se vinculan la vida ciclística.

Esta investigación no se termina aquí, nuestro objetivo es seguir indagando y buscando más información, donde a la vez otras personas se vinculen al intercambio de ideas enfocada al perfeccionamiento de una escuela para la formación integral de los ciclista, la cual sean capaz de enfrentar el reto de la vida diaria y permitiéndose un eslabón más en sus vidas.

\section{Agradecimientos}

Al mérito de la hermosa isla, Revolucionaria, comunista, Socialista Cuba, que ha dado a varias personas del mundo la oportunidad de cumplir sus sueños y de ser alguien en la vida, un país que siempre tendrá las puertas abiertas para todos los países hermanos.

Una retribución especial al personal y dirigentes del INDER. 


\section{INSTITUTO DE INVESTIGACIÓN CIENTÍFICA Y DESARROLLO TECNOLÓGICO \\ INCYT - UPSE}

A todos los profesores, dirigentes de la Universidad de Ciencias de la Cultura Física y el Deporte. Y, en especial, al Doctor Omar Paulas, que siempre estuvo en los momentos más difíciles para apoyarme.

Un reconocimiento a todos los atletas, entrenadores, mecánicos, dirigentes y trabajadores del velódromo "Reinaldo Paseiro".

A los profesores, trabajadores y dirigentes de la Escuela Internacional de Educación Física y Deporte.

\section{Referencias}

[1] Addines Fernández, Fátima (1998): La estrategia como resultado científico. Centro de Estudios Científicos de la Educación Universitaria de Camagüey, Camagüey.

[2] Brian Quinn, J. (1995): "Estrategias para el cambio". En Biblioteca de Planificación Estratégica. Ed: Prentice- Hall Hispanoamericana, S.A. México. P12.

[3] Campo R, Restrepo M. (1999): "Formación integral en la universidad y la acción de la Facultad de Educación de la Pontificia Universidad Javeriana". Revista digital Avanzada Universidad de Medellín, Medellín.

[4] Castellanos, D., B. Castellanos, M. J. Llivina y M. Silverio (2001): Hacia una concepción del aprendizaje desarrollador Centro de Estudios Educacionales del ISP “Enrique José Varona”. La Habana, P. 32

[5] Castro Ruz Fidel (2006): 3 de Octubre del 2000. Fidel y el Deporte. Editorial Deportes. Ciudad de la Habana.

[6] Colectivo de autores (junio 15, 2007): FORMACIÓN INTEGRAL. Universidad Javeriana. Veracruzana, Fines de formación integral.

Universidad.http://www.uv.mx/universidad/docto sofi/nme/finesfi.html. Veracruzana.

[7] Del Toro, M. (1968): Pequeño Larousse ilustrado. Ed: Revolucionaria. Instituto del libro. Ciudad de la Habana, P.441.

[8] Díaz M. (1998): Modernización curricular y alternativas pedagógicas. En: La formación académica y la práctica pedagógica. Documento. Cali; Universidad del Valle, Cali

[9] Ecuador - MSN Encarta. MSN Encarta (Mayo 18, 2007): Online Encyclopedia, Dictionary, Atlas, and Homework. http://encarta.msn.com/encyclopedia_761565312/ Ecuador.html Copyright Microsoft Encarta.

[10] Fernández, Lázaro (2007, Junio): Organización y funcionamiento de escuelas deportivas: http://www.mailxmail.com/curso/vida/escuelasde portivas_co.mht.

[11] Fichte (15 de junio 2007): La formación integral como eje fundamental en la capacitación profesional. Documento digital: http://www.integracion.com/formacion_coeje.mht

[12] Fines de formación integral (noviembre, 2007): Titulo del sitio: Universidad Veracruzana. Dirección:

http://www.uv.mx/universidad/doctosofi/nme/fin esfi.html Fecha de consulta.

[13] García Batista Gilberto y coautores (2004): Un profesional Imprescindible: El Maestro. Temas de Introducción a la Formación Pedagógica. Editorial Pueblo y Educación. Ciudad de la Habana.

[14] García Batista Gilberto y coautores (2004): Vivimos aprendiendo. Temas de Introducción a la Formación Pedagógica. Editorial Pueblo y Educación. Ciudad de la Habana. p 357.

[15] González Silva Humberto J. (2007): Colombia: La formación integral como objeto de la educación:

http://www.mailxmail.com/curso/formac integarl.co.mht.

[16] Martí J. (1961) "Escuela de electricidad" La América Nueva York, noviembre de 1883, en Ideario Pedagógico, imprenta Nacional de Cuba, La Habana, p120.

[17] Menguzzato y Renau. (1997): La Dirección Estratégica en la empresa. Un enfoque innovador del management. Libro reproducido por el MES. La Habana, P.78

[18] M Ordaz Lorenzo, R. Z(2003): La modelación como método científico general del conocimiento y sus potencialidades en el campo de la educación. ISPEJV. Material digitalizado. La Habana.

[19] Neuner, G. y otros (1981): Pedagogía. Ed: Libros para la Educación. La Habana, p 91

[20] Paula Chica, Maritza Gisella (2009): Introducción. Trabajo de en opción al título de Máster en Administración y Gestión de la Cultura Física y Deportes. Diagnostico de la Formación Integral de los ciclistas de alto rendimiento Deportivo en la ciudad de Guayaquil, Ecuador. Ciudad de la Habana, p 57.

[21]Paula Chica, Maritza Gisella (2006): Introducción. Trabajo de Diploma en opción al título de Licenciada en Educación Física y Deporte. Sistema de Capacitación para los entrenadores de ciclismo en la ciudad de Guayaquil, Ecuador. Ciudad de la Habana, p 57.

[22] Paulas, Omar (2007): Conferencia magistral en la segunda Convención Internacional de Ciencias de 
Actividad Física y Deporte "AFIDE". Administración y Gestión de las Organizaciones Deportivas. La Habana.

[23] Paulas, Omar (2007): Ponencia por comisiones en la segunda Convención Internacional de Ciencias de Actividad Física y Deporte "AFIDE". Desempeño profesional de los profesores de Educación Física. La Habana.

[24]Pérez, García, Águeda M, Norberto Valcárcel Izquierdo, Julia Añorga Morales (1997): La Educación Avanzada como en la Escuela cubana pedagógica. Soporte digital (s/e) (s/c)

[25] Ponce, Aníbal (1961): La Educación en la Comunidad Primitiva. Educación y Lucha de Clases. República de Cuba. Ministerio de Educación. Imprenta Nacional de Cuba. p 294.

[26]Quinn, J. B (1995b): Cambio estratégico: "El incrementalismo lógico". En: Biblioteca de Planificación Estratégica. Ed: Prentice- Hall Hispanoamericana, S.A. México. D.F.

[27] Ruiz, A. (1989): Metodología de la enseñanza de la Educación Física. Editorial Pueblo y Educación. Ciudad de la Habana, 2T

[28] Sierra Salcedo, Regla Alicia (2002): Modelación y estrategia: Algunas consideraciones desde una perspectiva pedagógica. Ed. Pueblo y Educación, Ciudad de La Habana, p. 317, 319.

[29] Sierra, R.A. (2000): La estrategia en instituciones educativas: criterios metodológicos para su diseño e implementación. Material impreso. ISPEJV. La Habana.

[30] Tijonov, A.N. (2003): Enciclopedia de Matemática. Tomado de Ordaz Lorenzo, R. La modelación como método científico general del conocimiento y sus potencialidades en el campo de la educación. ISPEJV. Material en soporte magnético.

[31]UCI Academy: (abril 20, 2007): Riders Certificate. Union Cycliste internationale. http://www.uciacademy.org/page.asp?pid=78\&id $=117$ 


\section{Modelos de Escuelas de Ciclismo Actuantes en Europa y Latinoamérica}

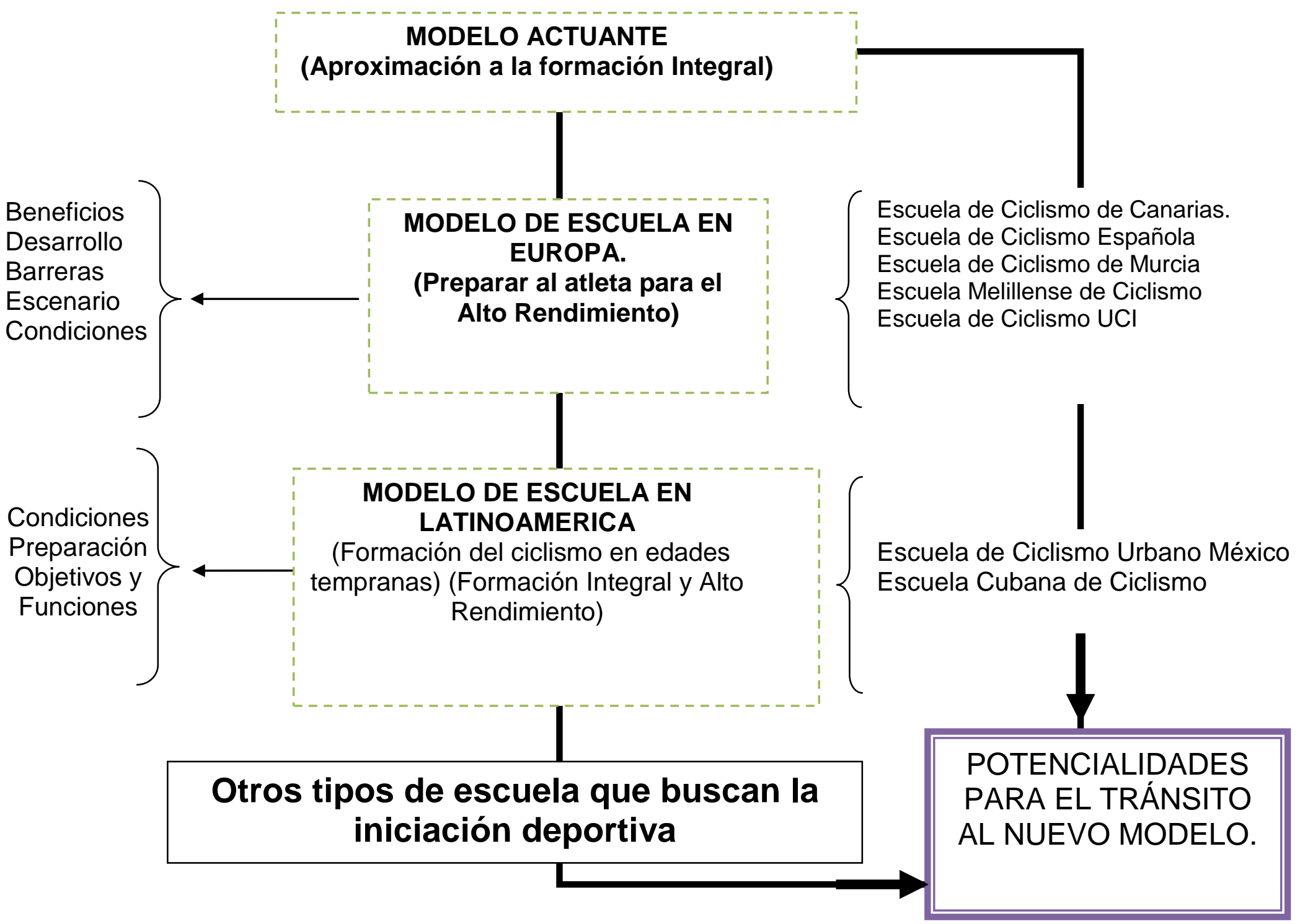


Modelo de Escuela de Ciclismo proyectivo para la Formación Integral de los Ciclistas de Alto Rendimiento Deportivo en Guayaquil-Ecuador.

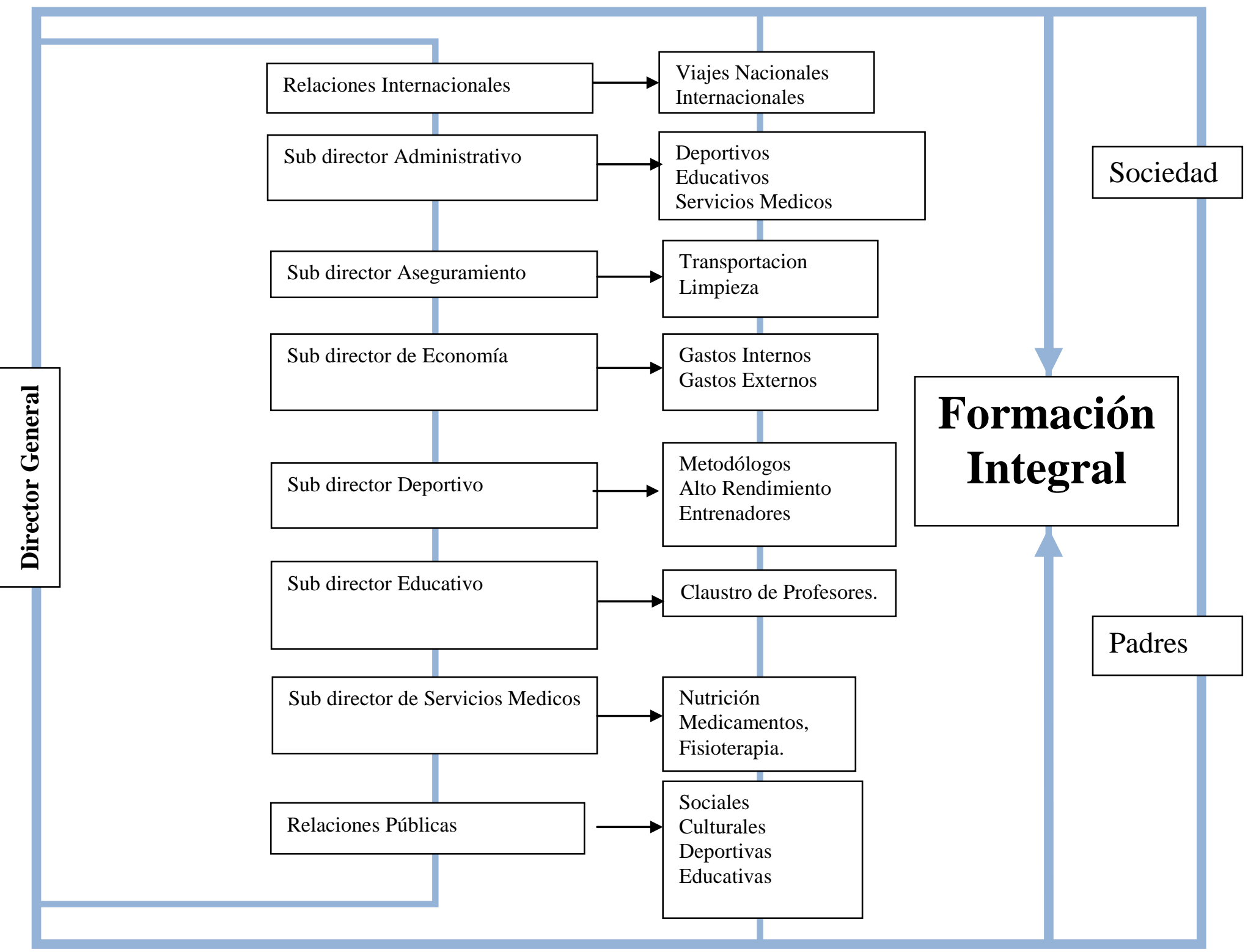

